\title{
Effectiveness of a Mobile Short-Message- Service-Based Disease Outbreak Alert System in Kenya
}

\author{
Mitsuru Toda, Ian Njeru, Dejan Zurovac, \\ Shikanga O-Tipo, David Kareko, \\ Matilu Mwau, Kouichi Morita
}

We conducted a randomized, controlled trial to test the effectiveness of a text-messaging system used for notification of disease outbreaks in Kenya. Health facilities that used the system had more timely notifications than those that did not $(19.2 \%$ vs. $2.6 \%)$, indicating that technology can enhance disease surveillance in resource-limited settings.

$\mathrm{O}$ utbreaks of epidemic diseases pose serious public health risks (1). Kenya, like other Africa countries, lacks the means to deliver adequate healthcare services. This weakness compromises the success of the World Health Organization's Integrated Disease Surveillance and Response (IDSR) and International Health Regulations (IHR) strategies and often results in incomplete, delayed, and poor-quality (i.e., not following standard case definitions in the IDSR guidelines) paper-based reporting from health facilities in remote areas. Furthermore, inadequate reporting limits health managers' ability to take appropriate and timely action in response to health events $(2,3)$.

Widespread expansion of mobile phone coverage in Africa (4) offers opportunities to overcome weaknesses in health systems and to improve medical and public health practice through mobile health (mHealth) (5). Despite many mHealth projects undertaken in Africa, their effectiveness has rarely been rigorously evaluated, limiting evidence-based policy adoptions or project expansion in scope or geography (6-9). In particular, evidence of effectiveness of mHealth interventions for enhancing disease surveillance is scarce (10). We undertook a clustered, randomized, controlled trial with 135 health facilities in Busia and Kajiado Counties in Kenya during November 2013-April 2014 to test the effectiveness of a mobile short-message-service (SMS)-based disease

Author affiliations: Japan International Cooperation Agency, Tokyo, Japan (M. Toda); Nagasaki University Institute of Tropical Medicine, Nagasaki, Japan (M. Toda, K. Morita); Ministry of Health Kenya, Nairobi, Kenya (I. Njeru, S. O-Tipo, D. Kareko); Kenya Medical Research Institute Wellcome Trust Research Programme, Nairobi (D. Zurovac); Oxford University, Oxford, UK (D. Zurovac); Kenya Medical Research Institute, Nairobi (M. Mwau)

DOI: http://dx.doi.org/10.3201/eid2204.151459 outbreak alert system (mSOS) for reporting immediately notifiable diseases.

\section{The Study}

mSOS is a formatted text-messaging system that enables communications between healthcare facility workers and Ministry of Health managers and uses a Web-based portal to monitor disease notifications and response actions taken by health managers (Figure 1; online Technical Appendix, http://wwwnc.cdc.gov/EID/article/22/4/151459-Techapp1.pdf). In our trial, health workers used mSOS for 6 months to send information about suspected cases or health events that required notification within 24 hours. Twelve diseases and conditions were selected for the study (online Technical Appendix Table 1). Before mSOS was implemented, we conducted a 1-day refresher training course on IDSR for in-charges (i.e., medical officers in charge) of 135 participating health facilities; the training focused on case definitions of notifiable diseases and on paper-based reporting. During the training, facilities were randomized into intervention and control groups; the intervention group received an additional day of training on mSOS. Paper-based reporting continued throughout the study period for both groups, so the intervention group would report cases 2 ways.

Our primary outcome was determining how many of the cases that required immediate notification were reported within the time specified. Our secondary outcome was determining, from among the cases for which notifications were sent, the proportion for which response actions were taken. For evaluation purposes, data from health facilities were collected for 6 -month periods before and after the intervention launch (i.e., IDSR and mSOS training and use of mSOS for 6 months). Cases detected, notifications submitted, and responses undertaken were extracted from facility records in both study groups. Notifications sent by SMS were retrieved from the mSOS system. Our primary analysis was intention-to-treat (i.e., analysis of cases from all health facilities as they were randomized, regardless of intervention exposure). Our secondary analysis was perprotocol (i.e., our trial protocol) and was restricted to cases reported by facilities whose in-charges had received training (i.e., IDSR training for control group; IDSR and $\mathrm{mSOS}$ training for intervention group; Figure 2).

Characteristics of health facilities and in-charges were similar; data from preintervention and postintervention 


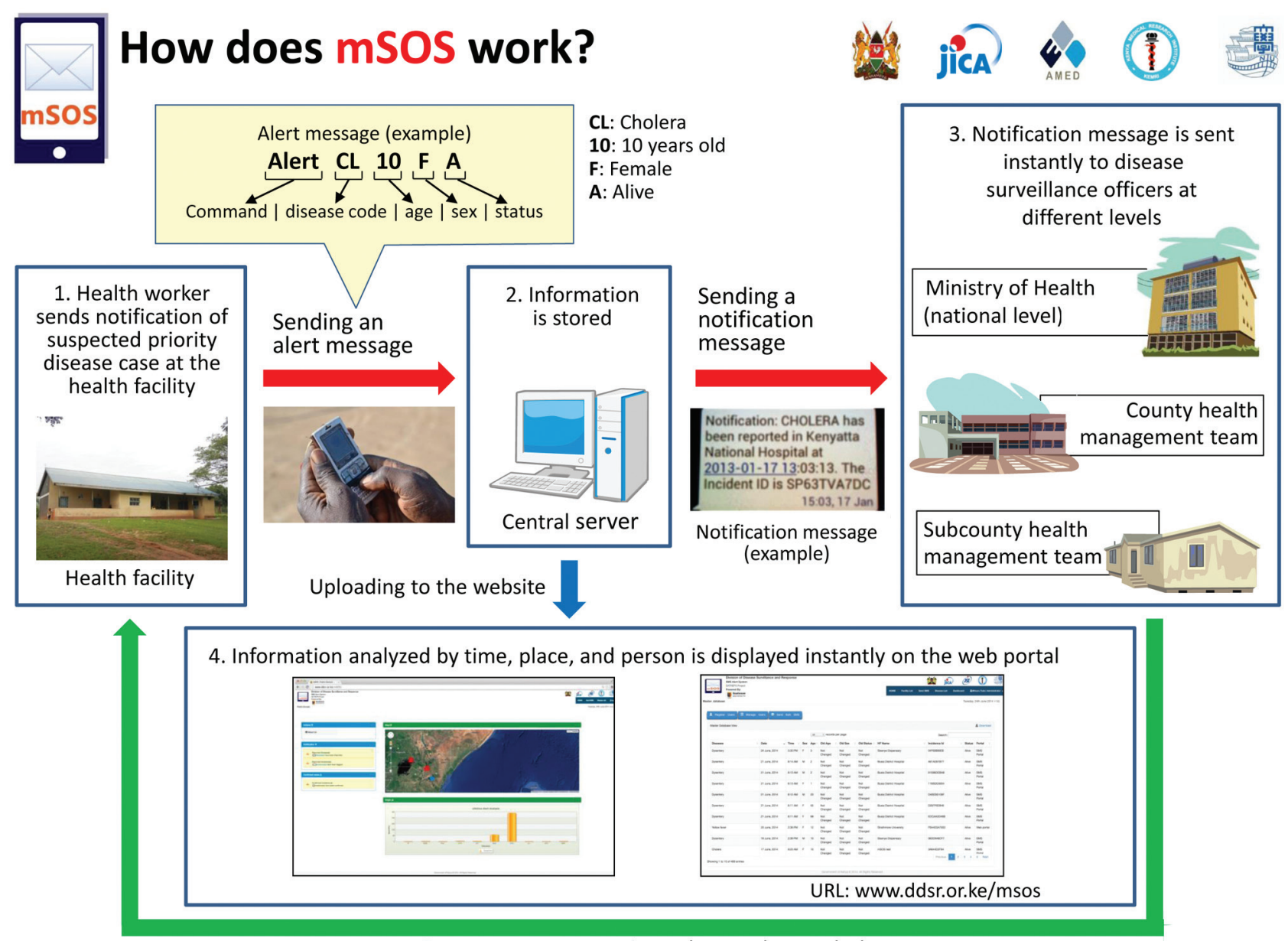

Prompt response action taken and recorded

Figure 1. Structure and communication flow of a mobile short-message-service-based disease outbreak alert system (mSOS) in Kenya. Source: mSOS Technical Working Group, Ministry of Health Kenya.

surveys showed no significant differences between control and intervention groups (Table 1). Follow-up surveys conducted 6 months after the intervention showed that $34(51.6 \%)$ of 66 intervention group in-charges received mSOS and IDSR training and $32(49.2 \%)$ of 65 control group in-charges received IDSR training (Figure 2; online Technical Appendix).

A retrospective review of the baseline (preintervention) surveys showed that 36 cases (19 for intervention group, 17 for control group), all measles, required immediate notification. Of these 36 cases, only 1 immediately notifiable case was reported (from a control facility using paper forms). During the 6-month period after the intervention, 169 immediately notifiable cases (130 for the intervention group, 39 for the control group) were detected: 160 measles, 6 anthrax, 2 Q fever, and 1 guinea worm. Of the 39 cases detected in the control group, notification of only 1 case $(2.6 \%)$, which was measles, was sent. Of the 130 immediately notifiable cases detected in the intervention group, $25(19.2 \%)$ were reported to disease surveillance coordinators at the subcounty, county, and national levels. This proportion of cases reported was significantly higher than that reported by the control group (\% difference 16.7 , 95\% CI 2.71-25.07; Table 2).

All 25 cases for which notifications were sent from the intervention group were measles cases reported through mSOS; 2 cases were also reported with paper forms. For these $25 \mathrm{mSOS}$ notifications, the threshold for a measles outbreak response ( 5 suspected cases) was met once, and disease surveillance coordinators at the subcounty level responded to this event. Furthermore, $24(96 \%)$ of the 25 suspected measles cases were reported within 24 hours.

In the per-protocol analysis, the percentage of cases for which notification was sent was greater in the intervention group than in the control group (27.3\% vs. $4.8 \%)$, but the difference was of borderline statistical significance $(\%$ difference $22.5,95 \%$ CI -0.32 to 34.13 by Wilson procedure with continuity correction [11]). Similar differences were found when the analysis was restricted to health 


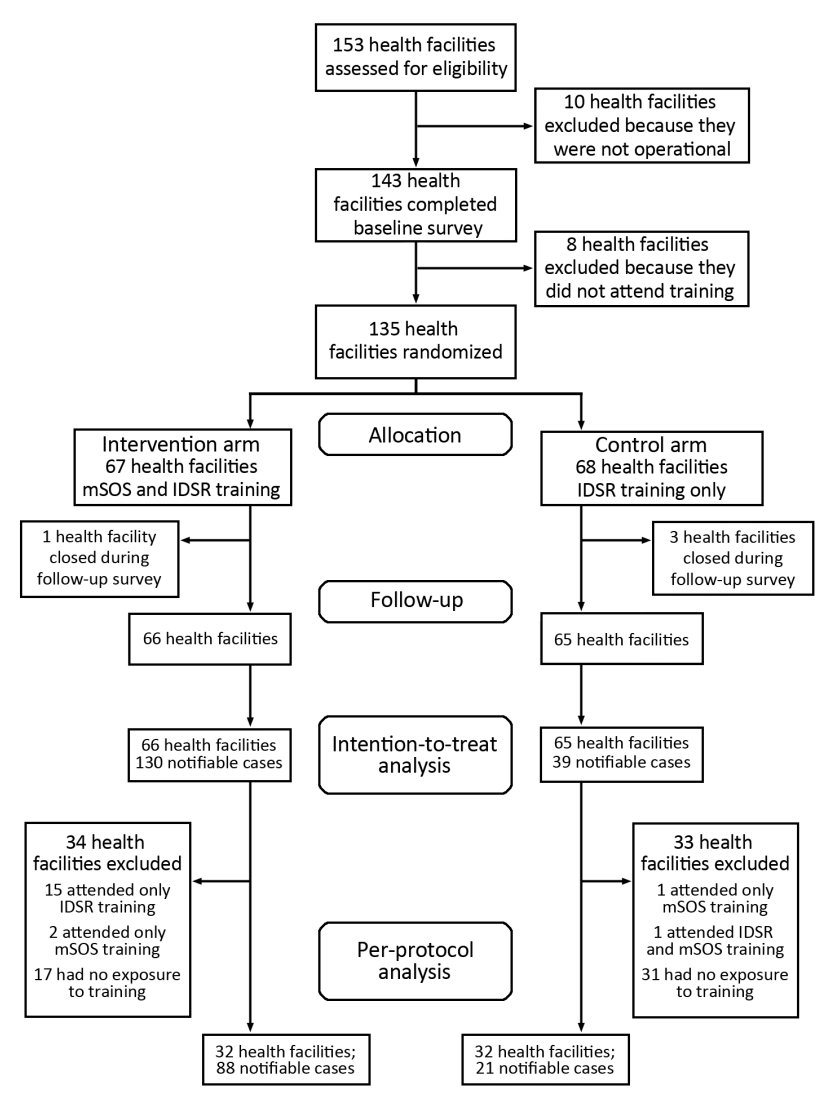

Figure 2. Profile of control and intervention health facilities and exclusions during the course of a study of a mobile shortmessage-service-based disease outbreak alert system (mSOS) in Kenya. IDSR, Integrated Disease Surveillance and Response.

facilities that stocked paper-based tools (i.e., control group, $1 / 18$ [5.6\%] vs. intervention group, $22 / 78$ [22.6\%]; \% difference $17.0,95 \%$ CI -2.93 to 35.30 ).

\section{Conclusions}

This study showed that SMS intervention significantly increased timely notifications; however, despite a relatively large improvement, response remained suboptimal, with timely notifications of only one fifth of detected cases. These findings mirror results of a study in Tanzania, which showed that SMS considerably increased vital registration coverage but fell far short of reporting actual birth and death events in the community (12).

Our study has implications for health managers who implement interventions to improve disease surveillance in resource-limited settings. First, the number of detected cases requiring immediate notification increased postintervention. This effect was observed in both intervention and control groups but was higher in the group using SMS; this group had a 7-fold increase in detected cases compared with baseline findings. IDSR refresher training may have contributed to increased case detection, and the combined interventions, including the technology component, resulted in a greater detection effect. Second, expecting health workers to complete paper-based forms and deliver them without incentive within 24 hours is ineffective for ensuring notification of cases, with or without exposure to the refresher training. Third, we observed a large drop-out rate $(47.4 \%)$ for health facility in-charges participating in the study. The study took place during a period of health management decentralization in Kenya, resulting in 47 new counties and in health worker transfers. Lack of on-the-job training for staff who did not attend the training and lack of support through posttraining follow-up and supportive supervision were weaknesses in the intervention. These systemic challenges, reported in other IDSR (13) and mHealth surveillance (14) projects, must be addressed to avoid compromising the sustainability of such interventions. Finally, attrition of health workers exposed to the intervention and lack of paper-based tools explain only part of our results. The short duration of the training deployed (15) and the possibly suboptimal quality of the training delivered (3) may have contributed to the unrealized full potential of the intervention.

Despite its limitations (online Technical Appendix), this study shows how technology in the form of mSOS can increase the rate of notifications of suspected disease outbreaks and enhance IHR compliance in resource-limited settings. Further investigation into ways to optimize the quality of delivery of mSOS interventions in countries with weak healthcare systems is justified.

\section{Acknowledgments}

We thank the Disease Surveillance and Response Unit at the Ministry of Health in Kenya and the Strathmore University Faculty of Information Technology for their tireless efforts to develop mSOS. We also thank the Kajiado and Busia County governments and the disease surveillance coordinators in the subcounties for their support. We are also grateful for field interviewers' work during the baseline and follow-up surveys and for statistical advice from Stella Karuri.

This study was supported by the Japan International Cooperation Agency and the Japan Agency for Medical Research and Development under the Science and Technology Research Partnerships project in Kenya (2012-2017). The study is published with the permission of the Director of KEMRI.

Ms. Toda is a public health specialist who focuses on health systems research in developing countries. She is currently a Japan International Cooperation Agency expert who provides technical support for strengthening disease surveillance at the Ministry of Health in Kenya. 
Table 1. Characteristics of health facilities and their in-charges for intervention and control groups and study periods, Kajiado County, Kenya*

\begin{tabular}{|c|c|c|c|c|c|}
\hline \multirow[b]{2}{*}{ Characteristic } & \multicolumn{2}{|c|}{ Preintervention, no. (\%) } & \multicolumn{3}{|c|}{ Postintervention, no. (\%) } \\
\hline & Control, $\mathrm{N}=65$ & Intervention, $\mathrm{n}=66$ & Control, $n=65$ & Intervention, $\mathrm{n}=66$ & $p$ value \\
\hline Health facilities, Kajiado County & $42(64.6)$ & $41(62.1)$ & $42(64.6)$ & $41(62.1)$ & 0.767 \\
\hline \multicolumn{6}{|l|}{ Ownership } \\
\hline Public & $39(60.0)$ & $45(68.2)$ & $39(60.0)$ & $45(68.2)$ & 0.329 \\
\hline Private & $15(23.1)$ & $13(19.7)$ & $15(23.1)$ & $13(19.7)$ & 0.637 \\
\hline FBO/NGO & $11(16.9)$ & $8(12.1)$ & $11(16.9)$ & $8(12.1)$ & 0.435 \\
\hline \multicolumn{6}{|l|}{ Level of care } \\
\hline Hospital/health center & $20(30.8)$ & $19(28.8)$ & $20(30.8)$ & $19(28.8)$ & 0.804 \\
\hline Dispensary & $40(61.54)$ & $43(65.15)$ & $40(61.5)$ & $43(65.2)$ & 0.668 \\
\hline Other facility & $5(7.7)$ & $4(6.1)$ & $5(7.7)$ & $4(6.1)$ & 0.712 \\
\hline \multicolumn{6}{|l|}{ Resource availability } \\
\hline Mobile phone & $65(100)$ & $66(100)$ & $65(100)$ & $66(100)$ & - \\
\hline Electricity & $45(69.2)$ & $47(71.2)$ & $54(83.1)$ & 49 (74.2) & 0.217 \\
\hline Water & $54(83.1)$ & 47 (71.2) & $51(78.5)$ & $50(75.8)$ & 0.713 \\
\hline Surveillance focal person & 48 (73.9) & $44(67.7)$ & $44(67.7)$ & 47 (71.2) & 0.662 \\
\hline IDSR reporting toolł & 22 (33.9) & $23(34.9)$ & $34(52.3)$ & $32(48.5)$ & 0.662 \\
\hline IDSR job aid & $44(67.7)$ & $44(66.7)$ & $49(75.4)$ & $55(83.3)$ & 0.261 \\
\hline \multicolumn{6}{|l|}{ Characteristic of in-charge } \\
\hline Female sex & $32(49.2)$ & $39(59.1)$ & $32(49.2)$ & $39(59.1)$ & 0.257 \\
\hline Median age, y (IQR)§ & $34(29-48)$ & $35(30-42)$ & $36(30-49.5)$ & $37(30-44)$ & 0.677 \\
\hline Doctor/clinical officer & $12(18.5)$ & $15(22.7)$ & $16(24.6)$ & $13(19.7)$ & 0.498 \\
\hline Nurse & $46(70.8)$ & $48(72.7)$ & $44(67.7)$ & $48(72.7)$ & 0.529 \\
\hline Other healthcare worker & $7(10.8)$ & $3(4.6)$ & $5(7.7)$ & $5(7.6)$ & 0.980 \\
\hline
\end{tabular}

*The table does not show data for Busia County because values will be inverse of data for Kajiado County (i.e., $\mathrm{N}$ minus $\mathrm{n}$ ). $\mathrm{N}=$ total facilities in both counties. The intervention group is the group of facility in-charges who were exposed to IDSR and mSOS training and to the mSOS intervention; the control group is the group of in-charges who were exposed to IDSR training only. FBO, faith-based organization; IDSR, Integrated Disease Surveillance and Response; in-charge, medical officer in charge of facility; IQR, interquartile range; NGO, nongovernment organization.

$+\mathrm{x}^{2}$ test was used to compare the proportions between control and intervention groups. Wilcoxon Mann Whitney test was used to compare medians between control and intervention groups (i.e., age of in-charges). Analyses were conducted by using an $\alpha$ level of 0.05 . The $p$ value is shown for the postintervention period only.

†Standardized IDSR paper-based reporting form for immediately notifiable diseases.

$\S$ Data are median and Interquartile range rather than numbers and percentages. Denominator excludes 3 facilities with missing values in the preintervention control group and 1 facility with missing values for each of the remaining 3 study groups.

Table 2. Postintervention reporting of immediately notifiable cases by study group under the intention-to-treat and per-protocol analysis* $^{*}$

\begin{tabular}{|c|c|c|c|c|c|}
\hline \multirow[b]{2}{*}{ Type of analysis } & \multicolumn{2}{|r|}{ Control } & \multicolumn{2}{|r|}{ Intervention } & \multirow[b]{2}{*}{ \% Difference $(95 \% \mathrm{Cl})$} \\
\hline & Total & Cases notified, no. (\%) & Total & Cases notified, no. (\%) & \\
\hline Intention to treat & 39 & $1(2.6)$ & 130 & $25(19.2)$ & $+16.7(2.71-25.07)$ \\
\hline Per protocol & 21 & $1(4.8)$ & 88 & $24(27.3)$ & $+22.5(-0.32$ to 34.13$)$ \\
\hline
\end{tabular}

*Intention-to-treat analysis indicates analysis of treatment groups as they were randomized, regardless of the intervention exposure; per-protocol analysis indicates restricted analysis of groups that completed the entire study according to the trial protocol.

\section{References}

1. Morse SS. Public health surveillance and infectious disease detection. Biosecur Bioterror. 2012;10:6-16.

2. Kasolo F, Yoti Z, Bakyaita N, Gaturuku P, Katz R, Fischer JE, et al. IDSR as a platform for implementing IHR in African countries. Biosecur Bioterror. 2013;1:163-9.

3. Phalkey RK, Yamamoto S, Awate P, Marx M. Challenges with the implementation of an Integrated Disease Surveillance and Response (IDSR) system: systematic review of the lessons learned. Health Policy Plan. 2015;30:131-43.

4. International Telecommunication Union. Measuring the information society report 2014. Geneva: Place des Nations; 2014.

5. World Health Organization. mHealth: new horizons for health through mobile technologies. Global observatory for eHealth series. Vol. 3. Geneva: The Organization; 2011.

6. Tomlinson M, Rotheram-Borus MJ, Swartz L, Tsai AC. Scaling up mHealth: where is the evidence? PLoS Med. 2013;10:e1001382. http://dx.doi.org/10.1371/journal.pmed.1001382

7. Free C, Phillips G, Galli L, Watson L, Felix L, Edwards P, et al. The effectiveness of mobile-health technology-based health behaviour change or disease management interventions for health care consumers: a systematic review. PLoS Med. 2013; 10:e1001362.

8. Free C, Phillips G, Watson L, Galli L, Felix L, Edwards P, et al. The effectiveness of mobile-health technologies to improve health care service delivery processes: a systematic review and metaanalysis. PLoS Med. 2013;10:e1001363. http://dx.doi.org/10.1371/ journal.pmed.1001363

9. Zurovac D, Talisuna AO, Snow RW. Mobile phone text messaging: tool for malaria control in Africa. PLoS Med. 2012;9:e1001176. http://dx.doi.org/10.1371/journal.pmed.1001176

10. Brinkel J, Kramer A, Krumkamp R, May J, Fobil J. Mobile phoneBased mHealth approaches for public health surveillance in subSaharan Africa: a systematic review. Int J Environ Res Public Health. 2014;11:11559-82. http://dx.doi.org/10.3390/ijerph111111559

11. Wilson EB. Probable inference, the law of succession, and statistical inference. J Am Stat Assoc. 1927;22:209-12. http://dx.doi.org/ 10.1080/01621459.1927.10502953

12. Kabadi G, Mwanyika H, de Savigny D. Innovations in monitoring vital events. Mobile phone SMS support to improve coverage of birth and death registration: a scalable solution. Herston (Australia): University of Queensland; 2013. 
13. Nsubuga P, Brown WG, Groseclose SL, Ahadzie L, Talisuna AO, Mmbuji P, et al. Implementing Integrated Disease Surveillance and Response: four African countries' experience, 1998-2005. Glob Public Health. 2010;5:364-80. http://dx.doi.org/10.1080/ 17441690903334943

14. Githinji S, Kigen S, Memusi D, Nyandigisi A, Wamari A, Muturi A, et al. Using mobile phone text messaging for malaria surveillance in rural Kenya. Malar J. 2014;13:107. http://dx.doi.org/10.1186/ 1475-2875-13-107
15. Sow I, Alemu W, Nanyunja M, Duale S, Perry HN, Gaturuku P. Trained district health personnel and the performance of integrated disease surveillance in the WHO African region. East Afr J Public Health. 2010;7:16-9.

Address for correspondence: Mitsuru Toda, Nagasaki University Institute of Tropical Medicine, 1-12-4 Sakamoto, Nagasaki, Japan; email: toda.mitsuru@tm.nagasaki-u.ac.jp

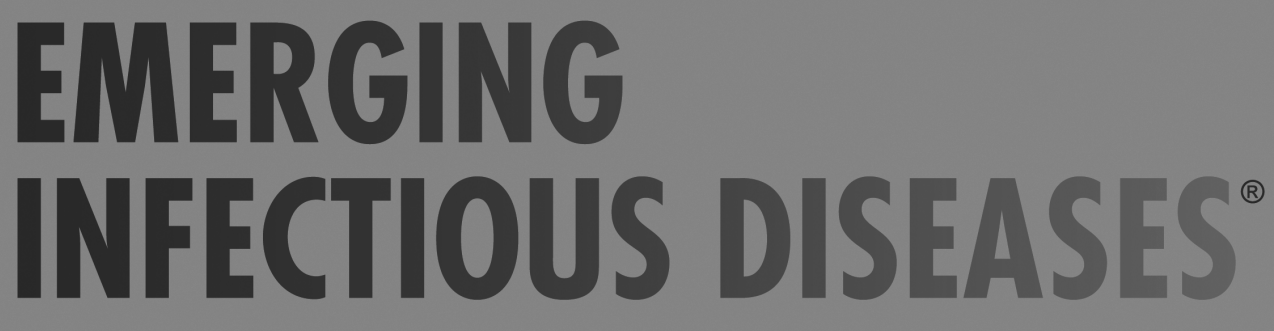

\section{JOURNAL BACKGROUND AND GOALS}

\section{What are "emerging" infectious diseases?}

Infectious diseases whose incidence in humans has increased in the past 2 decades or threatens to increase in the near future have been defined as "emerging." These diseases, which respect no national boundaries, include

$\star$ New infections resulting from changes or evolution of existing organisms.

$\star$ Known infections spreading to new geographic areas or populations.

$\star$ Previously unrecognized infections appearing in areas undergoing ecologic transformation.

$\star$ Old infections reemerging as a result of antimicrobial resistance in known agents or breakdowns in public health measures.

\section{Why an "Emerging" Infectious Diseases journal?}

The Centers for Disease Control and Prevention (CDC), the agency of the U.S. Public Health Service charged with disease prevention and health promotion, leads efforts against emerging infections, from AIDS, hantavirus pulmonary syndrome, and avian flu, to tuberculosis and West Nile virus infection. CDC's efforts encompass improvements in disease surveillance, the public health infrastructure, and epidemiologic and laboratory training.

Emerging Infectious Diseases represents the scientific communications component of CDC's efforts against the threat of emerging infections. However, even as it addresses CDC's interest in the elusive, continuous, evolving, and global nature of these infections, the journal relies on a broad international authorship base and is rigorously peer-reviewed by independent reviewers from all over the world.

\section{What are the goals of Emerging Infectious Diseases?}

1) Recognition of new and reemerging infections and understanding of factors involved in disease emergence prevention, and elimination. Toward this end, the journal

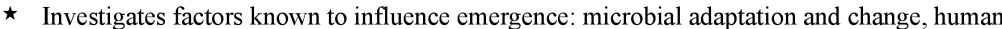
demographics and behavior, technology and industry, economic development and land use, international travel and commerce, and the breakdown of public health measures.

$\star$ Reports laboratory and epidemiologic findings within a broader public health perspective.

$\star$ Provides swift updates of infectious disease trends and research: new methods of detecting, characterizing, or subtyping pathogens; developments in antimicrobial drugs, vaccines, and prevention or elimination programs; case reports.

2) Fast and broad dissemination of reliable information on emerging infectious diseases. Toward this end, the journal

* Publishes reports of interest to researchers in infectious diseases and related sciences, as well as to public health generalists learning the scientific basis for prevention programs.

$\star$ Encourages insightful analysis and commentary, stimulating global interest in and discussion of emerging infectious disease issues.

* Harnesses electronic technology to expedite and enhance global dissemination of emerging infectious disease information. 